atrophy and intracellular accumulations of tau, TDP-43 or FUS protein. With the exception of a small number of familial forms the causes for FTLD are unknown. With our plasma proteomics approach we identified novel disease pathways that may open possibilities for treatment and diagnostics. Methods: We employed medium scale plasma proteomics, measuring more than 600 soluble cellular communication factors in plasma from control $(\mathrm{n}=83)$ and FTLD $(\mathrm{n}=92)$ patients with semantic dementia, as well as from FTLD patients with a familial Progranulin mutation $(\mathrm{n}=$ 24). We are using an antibody microarray based approach in combination with statistical and computational tools to identify altered pathways. Results: Our analysis revealed a deregulation of inflammatory markers, most prominently in the TNF-alpha and IL-1 pathways. We further confirmed an increase in TNF-alpha in the plasma of FTLD patients by ELISA. Currently we are in the process of analyzing the immune phenotype of FTLD patients and comparing it to healthy controls. Conclusions: Our results show evidence for a novel immune phenotype in FTLD patients with TDP-43 pathology. This opens up possibilities for a targeted anti-inflammatory therapy for this disease.

\section{FEATURED RESEARCH SESSIONS: F1-01 TACKLING OVERLAP OF NEUROPSYCHIATRIC SYMPTOMS IN ALZHEIMER'S AND OTHER DEMENTIAS: TOWARD A UNIFIED APPROACH TO EVALUATION AND TREATMENT}

\section{F1-01-01 EVIDENCE SUPPORTING USE OF NON- PHARMACOLOGIC INTERVENTIONS FOR NPS: WHERE DO WE GO FROM HERE?}

Carol Whitlatch, University of Michigan, Ann Arbor, Michigan, United States.

Background: This presentation will describe the evidence supporting nonpharamacologic interventions for NPS, suggest potential treatment mechanisms, and provide recommendations for future research. A review of 26 meta-analyses published between 2001 to 2011 and summarizing 518 studies of nonpharmacologic treatments suggests limited but promising evidence. Two basic approaches have been tested: non-specific, generalized programs (e.g., therapeutic activity, caregiver training, environmental redesign) to prevent or minimize NPS overall; and problem-solving (4-D approach) to eliminate, reduce or prevent targeted behaviors. However, research on both approaches remains flecked by imprecision including lack of standardized implementation and poor characterization of treatments, inattention to mechanisms of action, poor characterization of samples concerning dementia-type and disease stage, and reliance on subjective outcome measures. Proposed is the need for multi-site, large scale clinical trials to test the most promising nonpharmacologic approaches and which better characterize and link interventions, samples and presenting NPS. Future research must also contend with harmonizing NPS measures for comparative purposes, specifying nonpharmacologic strategies by dementia-type and behavior cluster, determine start and stop rules for using nonpharmacologic approaches, and evaluate mechanisms for integrating this approach in standard care.

\section{F1-01-02 TACKLING OVERLAP OF NEUROPSYCHIATRIC SYMPTOMS IN ALZHEIMER'S AND OTHER DEMENTIAS: TOWARD A UNIFIED APPROACH TO EVALUATION AND TREATMENT}

Constantine Lyketsos, Johns Hopkins University, Baltimore, Maryland, United States.

Background: Neuropsychiatric symptoms (NPS), while nearly universal in $\mathrm{AD}$, tend to occur in phenotypic clusters, which may reflect distinct pathophysiologies arising from specific underlying brain damage. Therefore, treatments targeting their specific cause(s) are required. For example, symptom clusters ("syndromes") characterized as affective, psychotic, agitated, apathetic, or reflecting sleep-wake cycle disorders have been proposed. Individual symptoms may co-occur in more than one syndrome at a given time, and these relationships may differ across the MCI to severe AD spectrum. Adding to the complexity, many NPS are thought to be the short-term result of environmental provocations (reactive psychological states and behaviors) which partly arise from non-specific effects of brain damage. For example, striking out during caregiving, acute nocturnal confusion, and excessively loud vocalization when confronted by cognitive limitations might be understood as the result of unsophisticated caregiving, acute delirium, or catastrophic reaction. The above issues have hampered the NPS field's ability to agree on how to evaluate, differentiate, and treat NPS. Gaining consensus on a common algorithmic approach to evaluation, differentiation, and how and when to initiate non-pharmacologic psychosocial treatments, would lay solid foundation for tackling NPS in the clinical world, and inform research in developing new therapies. We present an Expert Consensus Panel's proposed unified algorithm for the assessment, differentiation, and treatment of NPS in AD. Three talks are envisioned, followed by a panel discussion. The first talk uses epidemiologic findings from several international studies to define the challenge posed by NPS overlap, varying etiologies, and differences by AD stage, and lays the conceptual background for the proposed algorithm. The second talk expands the Four "D's" systematic approach to evaluation and differentiation (Rabins, Lyketsos, and Steele, 1999) that allows clinicians and researchers to develop patient specific interventions. The final talk presents evidence supporting the use of psychosocial interventions for NPS linked to the outcomes of the "4D" method, and discusses both specific and non-specific psychosocial approaches to the management of individual NPS syndromes. The latter assumes that such well articulated psychosocial approaches must be used prior to medication treatments. The panel concludes with speaker and audience discussion moderated by the panel chair.

\section{F1-01-03 THE 4-D APPROACH TO MANAGING NEUROPSYCHIATRIC SYMPTOMS}

Helen Kales, Benjamin Rose Institute on Aging, Cleveland, Ohio, United States.

Background: In real-world settings, few treatment options are currently available for neuropsychiatric symptoms (NPS) with the exception of common (and often off-label) use of psychotropic medications. While behavioral management strategies have been repeatedly shown to have significant benefits without the physical risks of medications, such strategies have received limited use in clinical settings. This talk will articulate a systematic approach to the evaluation and differentiation of NPS resulting from a Fall 2010 Expert Consensus Panel. This approach, expands upon the Four "Ds" (Rabins, Lyketsos, and Steele, 1999), and includes four stages: 1) Describe; 2) Decode; 3) Devise and; 4) Determine. First (Describe stage), the provider works with the caregiver to place the problem behavior into context. In the Decode stage, possible causes of the problem behavior are investigated. Next, (Devise stage), the provider creates a tailored treatment plan incorporating patient/caregiver preferences; this includes concurrently treating physical problems found via Decode and emphasizing a first-line use of behavioral strategies. In the final Determine stage, the provider and caregiver later assess the efficacy of Devise interventions. The talk will further detail how use of this systematic approach enables the creation of simple and effective behavioral interventions tailored to the patient, caregiver and environmental context.

FEATURED RESEARCH SESSIONS: F1-02 FEATURES OF STRUCTURAL AND FUNCTIONAL CONNECTIVITY IN NORMAL AGING

\section{F1-02-01 THE INFLUENCE OF COGNITIVE RESERVE ON INTER-INDIVIDUAL VARIABILITY IN RESTING-STATE CEREBRAL METABOLISM IN NORMAL AGING}

Christine Bastin ${ }^{1}$, Eric Salmon ${ }^{1}$, Mohamed Ali Bahri ${ }^{1}$, Brigitte Landau ${ }^{2}$, Andreas Fellgiebel $^{3}$, Dorothée Feyers ${ }^{1}$, Fabienne Collette ${ }^{1}$, Gaël Chetelat ${ }^{4}$, 